\title{
The role of atypical respiratory pathogens in exacerbations of chronic obstructive
} pulmonary disease

\author{
B.M.W. Diederen*, P.D.L.P.M. van der Valk ${ }^{\#}$, J.A.W.J. Kluytmans ${ }^{{ }^{\top},+}$, \\ M.F. Peeters ${ }^{\S}$ and R. Hendrix
}

ABSTRACT: The aetiology of acute exacerbations of chronic obstructive pulmonary disease (COPD) is heterogeneous and still under discussion. Serological studies have suggested that Mycoplasma pneumoniae, Chlamydia pneumoniae and Legionella pneumophila may play a role in acute exacerbations of COPD.

The presence of these atypical pathogens in sputum samples was investigated in patients with stable COPD and with acute exacerbations of COPD using real-time PCR. The present study was part of a randomised, double-blind, single-centre study and a total of 248 sputum samples from 104 COPD patients were included. In total, 122 samples obtained during stable disease (stablestate sputa) and 126 samples obtained during acute exacerbations of COPD (exacerbation sputa) were tested.

Of the 122 stable-state sputa, all samples were negative for M. pneumoniae and C. pneumoniae DNA, whereas one sample was positive for Legionella non-pneumophila DNA. Of the 126 exacerbation sputa, all samples were negative for $M$. pneumoniae and $C$. pneumoniae DNA, whereas one sample was positive for Legionella non-pneumophila DNA.

The possible relationship between the presence of atypical pathogens and the aetiology of acute exacerbations in chronic obstructive pulmonary disease was investigated in patients with stable disease and in those with acute exacerbations using real-time PCR. No indication was found of a role for Legionella spp., Chlamydia pneumoniae or Mycoplasma pneumoniae in stable, moderately severe chronic obstructive pulmonary disease and in its exacerbations.

KEYWORDS: Atypical pathogens, Chlamydia pneumoniae, chronic obstructive pulmonary disease, exacerbation, Legionella, real-time PCR

hronic obstructive pulmonary disease (COPD) is a major cause of morbidity and mortality in adults. According to the Global Burden of Disease Study [1], COPD is the fifth most common disease and the fourth leading cause of death in the world. The chronic course of this disease is frequently accompanied by acute exacerbations, characterised by an acute sustained worsening of the patient's condition from a stable state beyond normal day-to-day variations, which may warrant additional treatment [2]. Morbidity and mortality in COPD patients are, for the most part, related to acute exacerbations of COPD (AECOPD), which occur one to three times a year on average.

The aetiology of AECOPD is heterogeneous and still under discussion. For many years, there has been controversy regarding whether bacteria play a role in AECOPD, and thus, whether antibiotics play a role in disease management $[3,4]$. Several studies have shown an association between the presence of certain bacterial species, such as Streptococcus pneumoniae, Moraxella catarrhalis and Haemophilus influenzae, and AECOPD [3]. However, these potential pathogenic microorganisms (PPMO) were also present in sputa obtained from COPD patients with stable disease [5]. Apart from these bacterial PPMO, it was also shown that viral infections, with a prominent role for rhinoviruses, might trigger at least one-third of AECOPD [6]. Little is known about the presence of these viral PPMO in sputa obtained from COPD patients with stable disease. The term "atypical pathogen" most commonly refers to Mycoplasma pneumoniae, Chlamydia pneumoniae and Legionella pneumophila. The role of these bacteria in AECOPD remains unclear. Serological studies suggest that these atypical pathogens may play an important role in

\section{AFFILIATIONS}

*Regional Laboratory of Public Health Haarlem, Haarlem \#Dept of Pulmonary Medicine, Medisch Spectrum Twente, and ${ }^{f}$ Regional Laboratory of Public Health, Enschede,

- Laboratory for Microbiology and Infection Control, Amphia Hospital, Breda, and

+Dept of Medical Microbiology and Infection Control, VU Medical

University, Amsterdam

${ }^{\S}$ Regional Laboratory of Public Health Haarlem, Haarlem Laboratory for Medical Microbiology and Immunology, St Elisabeth Hospital, Tilburg, The Netherlands.

CORRESPONDENCE

B.M.W. Diederen

Regional Laboratory of Public Health

Haarlem

Boerhaavelaan 26

2035 RC

Haarlem

The Netherlands

Fax: 31135441264

E-mail: bramdiederen@gmail.com

Received:

February 032007

Accepted after revision:

April 122007

STATEMENT OF INTEREST

None declared.

European Respiratory Journal

Print ISSN 0903-1936

Online ISSN 1399-3003 
AECOPD [7-17]. However, the interpretation of the role of these atypical pathogens in AECOPD is not easy, since these organisms are difficult to cultivate from respiratory tract specimens. Also, variability among authors exists in the reliability and interpretation of the results of serological assays. Molecular diagnostic techniques, such as PCR, have become useful tools for the aetiological diagnosis of lower respiratory tract infections [18]. PCR can detect minute amounts of nucleic acids from potentially all PPMO; it does not depend on the viability of the target microbe; is probably less affected by previous antimicrobial therapy than culturebased methods are; and can provide results quickly. For patients with pneumonia, molecular techniques offer distinct advantages over conventional tests for the detection of $M$. pneumoniae, C. pneumoniae and Legionella spp. [18, 19].

The presence of these atypical pathogens in sputum samples was investigated in patients with stable COPD and those with AECOPD using real-time PCR.

\section{PATIENTS AND METHODS \\ Patients}

Patients were recruited in the period May 1999-March 2000 from the outpatient pulmonary clinic of the Medisch Spectrum Twente, a 1,150-bed teaching hospital in Enschede, the Netherlands, as previously described elsewhere $[5,20]$. Inclusion criteria were as follows. 1) A clinical diagnosis of stable COPD, as defined by the American Thoracic Society criteria. 2) No history of asthma. 3) No exacerbation in the month prior to enrolment. 4) Being a current or former smoker. 5) Age 40-75 yrs. 6) A baseline pre-bronchodilator forced expiratory volume in one second (FEV1) of $25-80 \%$ predicted. 7) A pre-bronchodilator ratio of FEV1 to inspiratory vital capacity value of $\leqslant 60 \%$. 8) A reversibility value of FEV1 $\leqslant 12 \%$ pred post-inhalation of $80 \mu \mathrm{g}$ of ipratropium bromide via a metered-dose inhaler with Aerochamber ${ }^{\circledR}$ (Boehringer Ingelheim, Alkmaar, the Netherlands). 9) A total lung capacity (TLC) $>$ TLC pred-1.64 $\times$ SD. 10) No maintenance treatment with oral steroids or antibiotics. 11) No medical condition with a low survival rate or serious psychiatric morbidity (e.g. cardiac insufficiency or alcoholism). 12) Absence of any other active lung disease (e.g. sarcoidosis). The hospital's medical ethical committee approved the present study. All patients provided written informed consent.

\section{Study protocol}

The present study was part of a randomised, double-blind, single-centre study, investigating the role of inhaled corticosteroids in COPD [20]. From the study, spontaneously expectorated sputum samples of patients were obtained at $0,4,7$ and 10 months in stable disease, and an additional sputum sample was collected at each hospital visit due to AECOPD. Clinically, exacerbations were defined as worsening of respiratory symptoms that resulted in the patient contacting the study office and receiving treatment by the study physician.

\section{Sputum samples}

Sputa were collected at scheduled visits to the outpatient department and at visits due to cases of exacerbation. Spontaneously expectorated sputum was collected in sterile vials and processed in the laboratory within $4 \mathrm{~h}$ of collection. Total sputum samples were homogenised by incubation at $37^{\circ} \mathrm{C}$ for 15 min with an equal volume of $0.1 \%$ dithiothreitol. Gram-stained sputum samples were examined microscopically and had to contain $<10^{5}$ epithelial cells $\cdot \mathrm{mL}^{-1}$ (i.e. $<1$ epithelial cell per high-power field) to be considered as representative bronchial samples. Polymorphonuclear cell count was not one of the criteria.

\section{DNA extraction}

$200 \mu \mathrm{L}$ of sputum was processed with the NucliSens ${ }^{\circledR}$ easyMAGIM) platform (bioMérieux, La Balme Les Grottes, France) with an elution volume of $50 \mu \mathrm{L}$, according to the manufacturer's instructions. $5 \mu \mathrm{L}$ of the eluate was used as a template in PCR. DNA was stored at $-20^{\circ} \mathrm{C}$ until PCR was performed.

\section{PCR assays}

For the detection of $C$. pneumoniae, an assay based on the nucleotide sequences of variable domain 2 and 4 of the outer membrane protein A gene of C. pneumoniae was used [21]. For the detection of Legionella, two separate assays were used targeted at specific regions within the 5S rRNA and the macrophage infectivity potentiator (mip) gene. The primers of the first Legionella spp. PCR-probe assay are based on the primers described by LINDSAY et al. [22], and detected in real time using a TaqMan probe Leg5S (Applied Biosystems, Nieuwerkerk a/d Ijssel, the Netherlands) [23]. The second PCR was a L. pneumophila-specific PCR based on the sequences of the mip gene [23]. For the detection of M. pneumoniae, an assay based on the P1 adhesin gene was used [24]. In short, primers M1 (forward 5'-GGT CAA TCT GGC GTG GAT CT-3') and M2 (5'-TGG TAA CTG CCC CAC AAG C-3') were used to obtain a 66 base pairs amplicon. Real-time detection was performed with a fluorescent (i.e. labelled with 6-carboxyfluorescein (FAM)) Taqman probe (5'-TCCCCC GTT GAA AAA GTG AGT GGG T-3'-FAM). Real-time PCR for all assays was performed on a ABi Prism ${ }_{\mathbb{R}} 7900 \mathrm{HT}$ Sequence Detection System (Applied Biosystems, Foster City, CA, USA). Results were expressed as threshold cycle $(\mathrm{Ct})$ values, corresponding to the cycle at which PCR enters the exponential phase. $\mathrm{Ct}$ values are proportional to the negative logarithm of the initial amount of input cDNA. If no increase in the fluorescent signal was observed after 50 cycles, the sample was assumed to be negative.

\section{RESULTS}

104 patients (median (range) age 63 (45-75) yrs; 86 males) provided a total of 248 sputum samples. 122 samples were obtained during stable disease (stable-state sputa) and 126 samples were obtained during AECOPD (exacerbation sputa). 76 patients provided both stable-state and AECOPD samples, 18 patients provided stable-state samples only and 10 patients provided AECOPD samples only. Since a substantial proportion of patients were unable to spontaneously expectorate an adequate sputum sample, and also because only microscopically representative sputum samples were used, the number of stable-state sputa is lower than theoretically expected. Of the 122 stable-state sputa, all samples were negative for $M$. pneumoniae and C. pneumoniae DNA, whereas one sample was positive for Legionella nonpneumophila DNA (positive in $5 \mathrm{~S}$ rRNA-based PCR; negative in mip gene-based PCR, Ct value $40)$. Of the 126 exacerbation sputa, all samples were negative 
for M. pneumoniae and C. pneumoniae DNA, whereas one sample was positive for Legionella nonpneumophila DNA (positive in 5S rRNA-based PCR, Ct value 43; negative in mip gene-based PCR). The Legionella-positive samples were obtained from different patients. In both Legionella-positive samples, S. pneumoniae was cultured to a level of growth of $>10^{5}$ colony-forming units $\cdot \mathrm{mL}^{-1}$.

\section{DISCUSSION}

The possible relationship between the presence of atypical pathogens and the aetiology of AECOPD in patients with stable COPD and in those with AECOPD was investigated using real-time PCR. No indication was found of a role for Legionella spp., C. pneumoniae or M. pneumoniae in COPD. Several potential contributions of bacterial infection to the aetiology, pathogenesis and clinical course of COPD can be identified. Regarding microbial patterns and their possible involvement in the aetiology of AECOPD, it is a common view that $H$. influenzae, S. pneumoniae and $M$. catarrhalis are the leading pathogens. Viruses have also been shown to cause acute exacerbations, frequently working as co-pathogens together with bacterial pathogens $[3,25]$. Recently, extensions of this concept have been provided. In several studies, serological evidence of C. pneumoniae, Legionella spp. and $M$. pneumoniae playing a role as a pathogen or co-pathogen in acute exacerbations has been reported [7-17]. One of the major issues not addressed in any of the sero-epidemiological studies is the correlation of serology with infection, as defined either by isolation of the organism in culture or by PCR. The lack of standardisation for the performance of serological test has made the interpretation of the published data from different laboratories about COPD patients difficult and, in the view of the present authors, debatable.

M. pneumoniae is a common cause of community-acquired pneumonia [2]. LIEBERMAN et al. [7] found M. pneumoniae to be the cause of AECOPD in $34(14 \%)$ out of 240 hospitalised patients, a higher value than those reported in other studies about the microbiological aetiology of AECOPD. In 24 (71\%) of these 34 patients, there was serological evidence of infection with at least one other respiratory agent in addition to $M$. pneumoniae. Patients that received antibiotics against $M$. pneumoniae did not have a better outcome in terms of a shorter hospital stay; in fact, the trend was actually in the opposite direction, with a mean length of hospitalisation for AECOPD that was 1.1 days less (5.3 versus 4.2 days). In three previous studies, M. pneumoniae was identified as a possible aetiological factor in $0.5-2.4 \%$ of AECOPD [26-28]. In a more recent study, SOLER et al. [29] did not find a single case of infection due to $M$. pneumoniae in a group of patients with severe COPD. $M$. pneumoniae is a fastidious organism, its culture is timeconsuming and it lacks sensitivity. Consequently, the laboratory diagnosis of $M$. pneumoniae infection has largely relied on commercially available serological tests, most often enzyme immunoassays, microparticle agglutination assays and the complement fixation test. However, few of these commercial assays have appropriate performances in terms of sensitivity and specificity and, therefore, some authors consider nucleic acid amplification the preferred diagnostic procedure for the diagnosis of M. pneumoniae infections [30].
In a study by LIEBERMAN et al. [9], 17\% of hospital admissions due to AECOPD showed serological evidence of acute infection with Legionella spp.. The present findings are in concordance with those of SOLER et al. [29], who observed no serological evidence for infection with L. pneumophila in a group of patients with severe COPD. An exhaustive review of AECOPD studies focused on the search of an aetiological agent by means of invasive procedures, such as bronchoscopic techniques, shows that Legionella spp. have never been identified using culture [2]. A disadvantage of serological testing is the inability to accurately detect all Legionella spp. and serogroups. Although seroconversion to L. pneumophila serogroup 1 is generally regarded as highly diagnostic, the sensitivity and specificity of the seroconversion to other species and serogroups have not been rigorously confirmed $[19,31]$. Legionella colonisation in COPD patients has never been detected and does not seem to be a risk factor for exacerbations in these patients. Of all pneumonia pathogens, Legionella non-pneumophila spp. probably presents the greatest risk for contamination measured in PCR, given the organism's ubiquitous environmental presence [18]. Owing to the high $\mathrm{Ct}$ values observed in both samples, amplification and detection of environmental Legionella DNA cannot be completely excluded.

C. pneumoniae has been reported to cause $4-16 \%$ of AECOPD, an observation based almost solely on serological evidence [14-17]. Studies of C. pneumoniae in COPD are complicated by several observations. Use of cell culture for detection of $C$. pneumoniae is technically demanding and time-consuming, and cell cultures generally have a low yield. As a consequence, the diagnosis of $C$. pneumoniae infection largely relies on serological testing using micro-immunofluorescence (MIF). The difficulties in C. pneumoniae serological testing are well known. Reports from different laboratories are highly variable, and adequate evaluations compared with a gold standard are lacking, which has led for calls for more standardised approaches in diagnostic testing [32]. Background rates of seropositivity by MIF can also be very high in some adult populations, sometimes $>80 \%$ [33]. In addition, smoking is associated with increased levels of serum antibodies to $C$. pneumoniae in patients with and without COPD, and serological conversion occurs even in the absence of symptoms [2, 21]. In 1988, it was reported that patients with coronary artery disease carry significantly more anti-C. pneumoniae immunoglobulin (Ig)G and IgA antibodies in their bloodstream than healthy controls [34]. Although initial reports were positive, more recent reports, often prospectively designed and adjusted for known cardiovascular risk factors, showed a negative or weak positive association overall between seropositivity for $C$. pneumoniae and atherosclerosis. Methodology has a strong impact on the possible association between C. pneumoniae and atherosclerosis: detection of the link between $C$. pneumoniae and coronary artery disease depends on the serological methodology chosen [35-37].

The present PCR results are in disagreement with those reported by BLASI et al. [38], which showed that C. pneumoniae DNA detection is associated with higher rates of exacerbation and airway microbial colonisation in patients with COPD. Of the 42 patients enrolled, individuals whose respiratory samples were C. pneumoniae DNA PCR positive (38\%) had a 
significantly greater number of pathogens on sputum culture than PCR negative patients. BLASI et al. [38] also found that C. pneumoniae-positive patients (in stable COPD) had a greater tendency towards frequent exacerbation, although this difference in exacerbation frequency between the two groups was small $\left(0.6\right.$ exacerbations $\left.\cdot \mathrm{yr}^{-1}\right)$. In a smaller, but similar, study SEEMUNGAL et al. [39] found no relationship between $C$. pneumoniae DNA detection in the airway at exacerbation and exacerbation frequency. The reported discrepancy in PCR positivity on respiratory samples between the present study and that of BLASI et al. [38] may be due to a number of reasons, including differences in PCR techniques used (real time instead of conventional, differences in DNA polymerases, decontamination with 2'-deoxyuridine-5'-triphosphate-uracilDNA glycosylase, the use of specific probes, inclusion of sufficient controls and the use of molecular grade water) [35, 36] and differences in study design and study population. In the current authors' view, real-time PCR is the current standard in the clinical microbiology laboratory, and the question of cross-contamination occurs whenever a nested PCR is performed. In this sense, the results of studies that use nested PCR to determine the prevalence of C. pneumoniae will always be questionable [40].

Only patients with less severe COPD (Global Initiative for Chronic Obstructive Lung Disease (GOLD) classification of severity stage I and II) were included and, therefore, the prevalence of atypical pathogens might be different in patients with more severe disease. However, this does not explain the differences found in the present study as compared with other serology-based studies. The current authors believe that the serological evidence of $C$. pneumoniae, Legionella spp. and $M$. pneumoniae playing a role as a pathogen or co-pathogen in AECOPD simply reflects the principal methodological problems of diagnosing such infections. From a statistical point of view, the use of a less specific diagnostic method for the detection of a pathogen will increase the likelihood of falsepositive reactions. Concern for serology specificity is even higher when a large percentage of other co-infections are also serologically identified [7-10].

Although it is difficult to draw general conclusions or even indications for standard care on the basis of the results of the present study, the current data provide indirect evidence against the clinical practice of prescribing antibiotics to patients with AECOPD. From the results of the present study it can be concluded that in patients with less severe COPD antibiotics directed at atypical pathogens are not necessary. However, prospective controlled trials are needed to really address the question of the effectiveness of antibiotic therapy directed against atypical respiratory pathogens and AECOPD.

In summary, in using real-time PCR to search for an association between the presence of atypical pathogens in patients with stable chronic obstructive pulmonary disease and in those patients with acute exacerbations of the disease, no indication was found of a role for Legionella spp., Chlamydia pneumoniae or Mycoplasma pneumoniae in stable, moderately severe chronic obstructive pulmonary disease and in its exacerbations. The present study indicates that nonstandardised serology might introduce a false association between atypical pathogens and acute exacerbations of chronic obstructive pulmonary disease.

\section{ACKNOWLEDGEMENTS}

The present authors would like to thank C. de Jong and I. Aarts (Laboratory of Medical Microbiology and Immunology, St Elisabeth Hospital, Tilburg, the Netherlands) for technical assistance.

\section{REFERENCES}

1 Murray CJ, Lopez AD. Alternative projections of mortality and disability by cause 1990-2020: Global Burden of Disease Study. Lancet 1997; 349: 1498-1504.

2 Burge S, Wedzicha JA. COPD exacerbations: definitions and classifications. Eur Respir J 2003; 21: Suppl. 41, 46S-53S.

3 Sethi S, Murphy TF. Bacterial infection in chronic obstructive pulmonary disease in 2000: a state-of-the-art review. Clin Microbiol Rev 2001; 14: 336-363.

4 Hirschmann JV. Do bacteria cause exacerbations of COPD? Chest 2000; 118: 193-203.

5 van der Valk P, Monninkhof E, van der Palen J, Zielhuis G, van Herwaarden C, Hendrix R. Clinical predictors of bacterial involvement in exacerbations of chronic obstructive pulmonary disease. Clin Infect Dis 2004; 39: 980-986.

6 Greenberg SB. Viral respiratory infections in elderly patients and patients with chronic obstructive pulmonary disease. Am J Med 2002; 112: Suppl. 6A, 28S-32S.

7 Lieberman D, Lieberman D, Ben-Yaakov M, et al. Serological evidence of Mycoplasma pneumoniae infection in acute exacerbation of COPD. Diagn Microbiol Infect Dis 2002; 44: 1-6.

8 Lieberman D, Dvoskin B, Lieberman DV, Kahane S, Friedman MG. Serological evidence of acute infection with the Chlamydia-like microorganism Simkania negevensis (Z) in acute exacerbation of chronic obstructive pulmonary disease. Eur J Clin Microbiol Infect Dis 2002; 21: 307-309.

9 Lieberman D, Lieberman D, Shmarkov O, et al. Serological evidence of Legionella species infection in acute exacerbation of COPD. Eur Respir J 2002; 19: 392-397.

10 Lieberman D, Lieberman D, Ben-Yaakov M, et al. Infectious etiologies in acute exacerbation of COPD. Diagn Microbiol Infect Dis 2001; 40: 95-102.

11 Ewig S. Legionella spp. in acute exacerbations of chronic obstructive pulmonary disease: what is the evidence? Eur Respir J 2002; 19: 387-389.

12 Lieberman D, Ben-Yaakov M, Lazarovich Z, Ohana B, Boldur I. Chlamydia pneumoniae infection in acute exacerbations of chronic obstructive pulmonary disease: analysis of 250 hospitalizations. Eur J Clin Microbiol Infect Dis 2001; 20: 698-704.

13 Hahn DL. Chlamydia pneumoniae, asthma, and COPD: what is the evidence? Ann Allergy Asthma Immunol 1999; 83: 271-288.

14 Blasi F, Legnani D, Lombardo VM, et al. Chlamydia pneumoniae infection in acute exacerbations of COPD. Eur Respir J 1993; 6: 19-22.

15 Beaty CD, Grayston JT, Wang SP, Kuo CC, Reto CS, Martin TR. Chlamydia pneumoniae, strain TWAR, infection in patients with chronic obstructive pulmonary disease. Am Rev Respir Dis 1991; 144: 1408-1410. 
16 Miyashita N, Niki Y, Nakajima M, Kawane H, Matsushima T. Chlamydia pneumoniae infection in patients with diffuse panbronchiolitis and COPD. Chest 1998; 114: 969-971.

17 Mogulkoc N, Karakurt S, Isalska B, et al. Acute purulent exacerbation of chronic obstructive pulmonary disease and Chlamydia pneumoniae infection. Am J Respir Crit Care Med 1999; 160: 349-353.

18 Murdoch DR. Molecular genetic methods in the diagnosis of lower respiratory tract infections. APMIS 2004; 112: 713-727.

19 Murdoch, DR., Diagnosis of Legionella infection. Clin Infect Dis 2003; 36: 64-69.

20 van der Valk P, Monninkhof E, van der Palen J, Zielhuis G, van Herwaarden $C$. Effect of discontinuation of inhaled corticosteroids in patients with chronic obstructive pulmonary disease: the COPE study. Am J Respir Crit Care Med 2002; 166: 1358-1363.

21 Tondella ML, Talkington DF, Holloway BP, et al. Development and evaluation of real-time PCR-based fluorescence assays for detection of Chlamydia pneumoniae. J Clin Microbiol 2002; 40: 575-583.

22 Lindsay DS, Abraham WH, Findlay W, Christie P, Johnston F, Edwards GF. Laboratory diagnosis of legionnaires' disease due to Legionella pneumophila serogroup 1: comparison of phenotypic and genotypic methods. J Med Microbiol 2004; 53: 183-187.

23 Diederen BM, de Jong CM, Marmouk F, Kluytmans JA, Peeters MF, van der Zee A. Evaluation of real-time PCR for the early detection of Legionella pneumophila DNA in serum samples. J Med Microbiol 2007; 56: 94-101.

24 Rossen JWA, Klein Breteler EG, Weersink AJ, Schuurman R, van Loon AM. Detection of respiratory pathogens by real-time PCR in clinical practice. Clin Microbiol Infect 2006; 12: Suppl. 4, O63.

25 Sethi S, Evans N, Grant BJ, Murphy TF. New strains of bacteria and exacerbations of chronic obstructive pulmonary disease. N Engl J Med 2002; 347: 465-471.

26 Busch RO, Saxtan D, Shultz PS, Finch E, Mufson MA. Infections with viruses and Mycoplasma pneumoniae during exacerbations of chronic bronchitis. J Infect Dis 1978; 137: 377-383.

27 Gump DW, Phillips CA, Forsyth BR, McIntosh K, Lamborn KR, Stouch WH. Role of infection in chronic bronchitis. Am Rev Respir Dis 1976; 113: 465-474.

28 Smith CB, Golden CA, Kanner RE, Renzetti AD Jr. Association of viral and Mycoplasma pneumoniae infections with acute respiratory illness in patients with chronic obstructive pulmonary diseases. Am Rev Respir Dis 1980; 121: 225-232.
29 Soler N, Torres A, Ewig S, et al. Bronchial microbial patterns in severe exacerbations of chronic obstructive pulmonary disease (COPD) requiring mechanical ventilation. Am J Respir Crit Care Med 1998; 157: 1498-1505.

30 Beersma MF, Dirven K, van Dam AP, Templeton KE, Claas EC, Goossens H. Evaluation of 12 commercial tests and the complement fixation test for Mycoplasma pneumoniae-specific immunoglobulin G (IgG) and $\operatorname{IgM}$ antibodies, with PCR used as the "gold standard". J Clin Microbiol 2005; 43: 2277-2285.

31 Roig J, Casal J. Is serological diagnosis of Legionnaires' disease a reliable method? Diagn Microbiol Infect Dis 2002; 43: 171-172.

32 Dowell SF, Peeling RW, Boman J, et al. Standardizing Chlamydia pneumoniae assays: recommendations from the Centers for Disease Control and Prevention (USA) and the Laboratory Centre for Disease Control (Canada). Clin Infect Dis 2001; 33: 492-503.

33 Hyman CL, Roblin PM, Gaydos CA, Quinn TC, Schachter J, Hammerschlag MR. Prevalence of asymptomatic nasopharyngeal carriage of Chlamydia pneumoniae in subjectively healthy adults: assessment by polymerase chain reaction-enzyme immunoassay and culture. Clin Infect Dis 1995; 20: 1174-1178.

34 Saikku P, Leinonen M, Mattila K, et al. Serological evidence of an association of a novel Chlamydia, TWAR, with chronic coronary heart disease and acute myocardial infarction. Lancet 1988; 2: 983-986.

35 Ieven MM, Hoymans VY. Involvement of Chlamydia pneumoniae in atherosclerosis: more evidence for lack of evidence. J Clin Microbiol 2005; 43: 19-24.

36 Maraha B, Berg H, Kerver M, et al. Is the perceived association between Chlamydia pneumoniae and vascular diseases biased by methodology? J Clin Microbiol 2004; 42: 3937-3941.

37 Hoymans VY, Bosmans JM, Van Renterghem L, et al. Importance of methodology in determination of Chlamydia pneumoniae seropositivity in healthy subjects and in patients with coronary atherosclerosis. J Clin Microbiol 2003; 41: 4049-4053.

38 Blasi F, Damato S, Cosentini R, et al. Chlamydia pneumoniae and chronic bronchitis: association with severity and bacterial clearance following treatment. Thorax 2002; 57: 672-676.

39 Seemungal TA, Wedzicha JA, MacCallum PK, Johnston SL, Lambert PA. Chlamydia pneumoniae and COPD exacerbation. Thorax 2002; 57: 1087-1088.

40 Apfalter P, Assadian O, Blasi F, et al. Reliability of nested PCR for detection of Chlamydia pneumoniae DNA in atheromas: results from a multicenter study applying standardized protocols. J Clin Microbiol 2002; 40: 4428-4434. 